\title{
Interventions for enhancing adherence to treatment in adults with bronchiectasis (Review)
}

McCullough A, Thomas ET, Ryan C, Bradley JM, O'Neill B, Elborn S, Hughes C

McCullough A, Thomas ET, Ryan C, Bradley JM, O'Neill B, Elborn S, Hughes C.

Interventions for enhancing adherence to treatment in adults with bronchiectasis.

Cochrane Database of Systematic Reviews 2015, Issue 11. Art. No.: CD011023.

DOI: 10.1002/14651858.CD011023.pub2.

www.cochranelibrary.com 
ABSTRACT 1

PLAIN LANGUAGE SUMMARY

BACKGROUND

OBJECTIVES

METHODS

RESULTS

Figure 1.

DISCUSSION

AUTHORS' CONCLUSIONS

ACKNOWLEDGEMENTS

REFERENCES

CHARACTERISTICS OF STUDIES

APPENDICES

WHAT'S NEW

CONTRIBUTIONS OF AUTHORS

DECLARATIONS OF INTEREST

SOURCES OF SUPPORT

DIFFERENCES BETWEEN PROTOCOL AND REVIEW

INDEX TERMS

\section{TABLE OF CONTENTS}


[Intervention Review]

\section{Interventions for enhancing adherence to treatment in adults with bronchiectasis}

Amanda McCullough1, Elizabeth T Thomas ${ }^{1}$, Cristin Ryan², Judy M Bradley33,4, Brenda O'Neill5, Stuart Elborn 4,6, Carmel Hughes 7

1Centre for Research in Evidence-Based Practice (CREBP), Bond University, Gold Coast, Australia. 2School of Pharmacy, Royal College of Surgeons in Ireland, Dublin 2, Ireland. ${ }^{3}$ The Wellcome Trust-Wolfson Northern Ireland Clinical Research Facility U Floor, Queen's University Belfast, Belfast, UK. ${ }^{4}$ Department of Respiratory Medicine, Belfast Health and Social Care Trust, Belfast, UK. ${ }^{5}$ Centre for Health and Rehabilitation Technologies (CHaRT), Institute of Nursing and Health Research, Ulster University, Newtownabbey, UK. ${ }^{6}$ Centre for Infection and Immunity, Queen's University Belfast, Belfast, UK. 7School of Pharmacy, Queen's University Belfast, Belfast, UK

Contact: Amanda McCullough, Centre for Research in Evidence-Based Practice (CREBP), Bond University, Gold Coast, Queensland, Australia. amandamccullough104@gmail.com,amccullo@bond.edu.au.

Editorial group: Cochrane Airways Group.

Publication status and date: Edited (no change to conclusions), published in Issue 8, 2017.

Citation: McCullough A, Thomas ET, Ryan C, Bradley JM, O'Neill B, Elborn S, Hughes C. Interventions for enhancing adherence to treatment in adults with bronchiectasis. Cochrane Database of Systematic Reviews 2015, Issue 11. Art. No.: CD011023. DOI: 10.1002/14651858.CD011023.pub2.

Copyright @ 2017 The Cochrane Collaboration. Published by John Wiley \& Sons, Ltd.

\section{A B S T R A C T}

\section{Background}

Bronchiectasis is characterised by a widening of the airways, leading to excess mucus production and recurrent infection. It is more prevalent in women and those in middle age. Many patients with bronchiectasis do not adhere to treatments (medications, exercise and airway clearance) prescribed for their condition. The best methods to change these adherence behaviours have not been identified.

\section{Objectives}

To assess the effects of interventions to enhance adherence to any aspect of treatment in adults with bronchiectasis in terms of adherence and health outcomes, such as pulmonary exacerbations, health-related quality of life and healthcare costs.

\section{Search methods}

We searched the Cochrane Airways Group Specialised Register (CAGR), which contains trial reports identified through systematic searches of CENTRAL, MEDLINE, EMBASE, CINAHL, AMED and PsycINFO, from inception to October 2015.

\section{Selection criteria}

We planned to include randomised controlled trials (RCTs) of adults with bronchiectasis that compared any intervention aimed at enhancing adherence versus no intervention, usual care or another adherence intervention. We excluded studies of those who had bronchiectasis due to cystic fibrosis.

\section{Data collection and analysis}

Two review authors (AMCC and ET) independently screened titles, abstracts and full-texts of identified studies.

\section{Main results}

Searches retrieved 36 studies reported in 37 articles; no eligible studies were identified. 


\section{Authors' conclusions}

We did not identify any studies that assessed the effect of interventions to enhance adherence to treatment in bronchiectasis. Adequately powered, well-designed trials of adherence interventions for bronchiectasis are needed.

\section{PLAIN LANGUAGE SUMMARY}

\section{How can adults with bronchiectasis be encouraged to take treatments as prescribed by their health professional?}

\section{Review question}

How can adults with bronchiectasis be encouraged to complete treatments such as medications, breathing exercises or other exercise as prescribed by their health professional?

\section{Background}

Bronchiectasis is caused by a widening of the airways. People with bronchiectasis have too much mucus in their lungs and often suffer from chest infections. Bronchiectasis occurs more commonly in women, and those in middle age. Treatments prescribed for bronchiectasis include inhalers, nebulisers and airway clearance physiotherapy. Doing all of these correctly can take up a lot of time every day. Many people with bronchiectasis find it difficult to fit these treatments into their daily lives.

Taking treatments in the way they have been prescribed by a health professional is called adherence. People with bronchiectasis who have low adherence to treatments like nebulised antibiotics have more chest infections than those with high adherence. We wanted to find out ways of encouraging adults with bronchiectasis to take treatments as prescribed by their health professional.

\section{Search date}

The evidence is current to October 2015.

\section{Key results}

We identified 37 reports but did not find any studies that tested ways to improve adherence to treatment. Properly-designed research studies that include an appropriate number of people with bronchiectasis are needed to answer this question. 


\section{B A C K G R O U N D}

\section{Description of the condition}

More than 600 million people worldwide suffer from chronic respiratory disease (WHO 2007), leading to 4.2 million deaths annually (WHO 2010). Bronchiectasis is characterised by chronic dilation of the airways, leading to excess sputum production and recurrent infection (Pasteur 2010). It is an under-diagnosed and under-investigated condition (Gibson 2013). Research into new treatments is urgently required to improve the health status of patients with this condition (Gibson 2013). The global prevalence of bronchiectasis is currently not known. The most recent prevalence study, conducted in the United States, demonstrated that prevalence of the condition is increasing at a rate of $8.7 \%$ annually, with an 8-year prevalence of 1106 per 100,000 of the population (Seitz 2012). Bronchiectasis-associated annual healthcare costs were estimated at US\$630 million in 2005 (Weycker 2005), and bronchiectasis-associated hospital admissions range between 2 and 6 per 100,000 in Europe (Gibson 2013). Given the recognised under-diagnosis of this condition, it is likely that prevalence and the associated healthcare burden are even greater than reported.

\section{Description of the intervention}

Patients with bronchiectasis are commonly prescribed a complex regimen of medication, airway clearance techniques and exercise (Pasteur 2010; Chang 2015). However, there is insufficient highquality evidence of their effectiveness (Franco 2003; Evans 2007; Pizzutto 2010; Lee 2013; Goyal 2014; Hart 2014; Wilkinson 2014) and no licensed therapies for the treatment of bronchiectasis. New therapies are being adapted and tested in patients with bronchiectasis (Altenburg 2013; Bilton 2013; Haworth 2013; Serisier 2013; Wilson 2013), and it is likely that the treatment burden for those with bronchiectasis will continue to grow as some of these become licensed treatments. This burden may lead to further reductions in adherence to treatment.

Adherence is defined as "the extent to which a person's behaviour - taking medication, following a diet and/or executing lifestyle changes - corresponds with agreed recommendations from a healthcare provider" (WHO 2003). Recent data suggest that as few as $53 \%$ of patients with bronchiectasis are adherent to medication and $41 \%$ to airway clearance (McCullough 2014a). This is important because those who are non-adherent to inhaled antibiotics have significantly more pulmonary exacerbations (McCullough 2014a) and a shorter time to first exacerbation (Haworth 2013). Interventions to enhance adherence are needed but little is known about the types of adherence interventions that have been used in bronchiectasis or which, if any, are effective.

\section{How the intervention might work}

Adherence is a complex behavioural process. Adherence interventions use educational, psychological and behavioural techniques, in isolation or in combination with each other, to alter adherence behaviour. However, education alone is insufficient to alter adherence behaviour (Haynes 2008). Enhanced adherence may lead to improved health outcomes, including reductions in pulmonary exacerbations and in healthcare costs for people with bronchiectasis. Patients may experience improved health-related quality of life as a consequence of enhanced adherence.

\section{Why it is important to do this review}

Low adherence is associated with more frequent pulmonary exacerbations for patients with bronchiectasis (McCullough 2014a). Therefore, enhancing adherence has the potential to lead to improved health outcomes in this patient population. Little is known about the types of adherence interventions that have been used in bronchiectasis and which, if any, are effective. We planned to analyse randomised controlled trials to determine the content of the interventions that have been tested in this population and their effectiveness in enhancing adherence and health outcomes.

\section{O B JECTIVES}

To assess the effects of interventions to enhance adherence to any aspect of treatment in adults with bronchiectasis in terms of adherence and health outcomes, such as pulmonary exacerbations, health-related quality of life and healthcare costs.

\section{METHODS}

\section{Criteria for considering studies for this review}

\section{Types of studies}

We planned to include randomised controlled trials (RCTs). We planned to include studies reported as full text, those published as abstract only and unpublished data. We also planned to include both parallel and cross-over group designs.

\section{Types of participants}

Adults (18 years of age or older) with bronchiectasis diagnosed by high-resolution computed tomography were eligible for inclusion. We planned to include both those who are acutely unwell and those who were stable. We excluded participants with bronchiectasis caused by underlying cystic fibrosis. We also planned to include studies that contained only a subset of relevant participants, where data were reported separately.

\section{Types of interventions}

We planned to include trials comparing any intervention aimed at enhancing adherence (including self management, education, service developments, reminders and other psychological and behavioural techniques) versus no intervention, usual care or another adherence intervention. We excluded studies that compared adherence between two different treatments without any adherence intervention (e.g. those comparing adherence to one type of medication, airway clearance technique, inhaler, mask or nebuliser versus another) and those that merely report adherence to treatment.

\section{Types of outcome measures}

Reporting in the trial one or more of the outcomes listed below was not an inclusion criterion for the review. We planned to include studies on the basis of type of study, participants and interventions. We planned to extract data collected at the end of the intervention period (i.e. after the last intervention) and at the end of the study follow-up (i.e. the end of the study), if different from data obtained at the end of the intervention period. Where appropriate, we also planned to extract data collected at interim time points (i.e. data collected at time points other than end of the intervention and end of the study). 


\section{Primary outcomes}

1. Adherence to at least one aspect of treatment (medication, airway clearance, medical devices or physical activity) as measured by direct (e.g. electronic monitoring, directly observed therapy) or indirect methods (e.g. self report, prescription refill data).

2. Rate of, duration of or time to first pulmonary exacerbation of bronchiectasis, defined according to the investigators' definition.

\section{Secondary outcomes}

1. Time to hospitalisation, number of hospital admissions or hospital days for a pulmonary exacerbation of bronchiectasis.

2. Pulmonary function measures (forced expiratory volume in one second $\left(F V_{1}\right)$, forced vital capacity (FVC), $\mathrm{FEV}_{1} / \mathrm{FVC}$, forced expiratory flow $(F E F)_{25-75}$, peak expiratory flow (PEF)).

3. Health-related quality of life measured using a generic or disease-specific tool (e.g. St George's Respiratory Questionnaire, Quality of Life Questionnaire-Bronchiectasis).

4. Exercise capacity as measured by any exercise capacity tool (e.g. six-minute walk test, incremental shuttle walk test).

5. Healthcare costs including costs of intervention, costs of devices and overall expenditures.

6. Any adverse events associated with adherence or nonadherence to treatment.

Adherence to treatment is a primary outcome of this review, as this is the key outcome that adherence interventions aim to change. Reducing the frequency, duration of or time to pulmonary exacerbations, minimising hospital admissions, improving healthrelated quality of life and maintaining pulmonary function are key clinical outcomes of bronchiectasis treatments (Pasteur 2010) and thus have been chosen as the clinical outcomes for this review. To judge whether interventions can be implemented in clinical practice, it is necessary to know the cost-effectiveness of the intervention. Finally, to ensure patient safety, it is important to be aware of any adverse events associated with the intervention.

\section{Search methods for identification of studies}

\section{Electronic searches}

We identified trials from the Cochrane Airways Group Specialised Register (CAGR), which is maintained by the Trials Search Coordinator for the Group. The Register contains trial reports identified through systematic searches of bibliographic databases including the Cochrane Central Register of Controlled Trials (CENTRAL), MEDLINE, EMBASE, CINAHL, AMED and PsycINFO, and by handsearching of respiratory journals and meeting abstracts (see Appendix 1 for further details). We searched all records in the CAGR using the search strategy provided in Appendix 2. We also conducted a search of ClinicalTrials.gov (www.ClinicalTrials.gov) and the World Health Organization (WHO) trials portal (www.who.int/ictrp/en/). We searched all databases from the time of their inception up to October 2015, and we imposed no restriction on language of publication.

\section{Searching other resources}

We planned to check reference lists of all primary studies and review articles for additional references. We planned to search relevant manufacturers' websites for trial information. We planned to search for errata or retractions from included studies published in full text on PubMed (www.ncbi.nlm.nih.gov/pubmed) and report within the review the date this was done.

\section{Data collection and analysis}

\section{Selection of studies}

Two review authors (AMCC and ET) independently screened titles and abstracts for inclusion of all studies identified as a result of the search and coded them as 'retrieve' (eligible or potentially eligible/ unclear) or 'do not retrieve'. We retrieved the full-text study reports/ publications, and two review authors ( $\mathrm{AMCC}$ and ET) independently screened the full text and identified studies for inclusion, and identified and recorded reasons for exclusion of ineligible studies. We resolved disagreements through discussion, or, if required, consulted a third review author $(\mathrm{CH})$. We identified and excluded duplicates and collated multiple reports of the same study, so that each study rather than each report was the unit of interest in the review. We recorded the selection process in sufficient detail to complete a PRISMA flow diagram and a Characteristics of excluded studies table.

\section{Data extraction and management}

We planned to document study characteristics and outcome data using a data collection form that had been piloted on at least one study in the review. Two review authors (AMCC and ET) planned to extract the following study characteristics from included studies.

1. Methods: study design, total duration of study, details of any 'run-in' period, number of study centres and locations, study setting, withdrawals and date of study.

2. Participants: number of participants, mean age, age range, gender, ethnicity, educational level, severity of condition, diagnostic criteria, baseline lung function, smoking history, inclusion criteria and exclusion criteria.

3. Interventions: description of the intervention including duration of run-in, intervention and follow-up; type of intervention (including its components (e.g. self management, education, service developments, reminders and other psychological and behavioural components), how and where it was delivered, by whom and its theoretical rationale) and type of control group.

4. Outcomes: primary and secondary outcomes specified and collected, and time points reported.

5. Notes: funding for trial and notable conflicts of interest of trial authors.

Two review authors (AMCC and ET) planned to independently extract outcome data from included studies. We planned to note in the Characteristics of included studies table if outcome data were not reported in a usable way. We aimed to resolve disagreements by consensus or by involving a third review author $(\mathrm{CH})$. One review author (AMCC) planned to transfer data into the Review Manager (RevMan 2014) file. We would have double-checked that data had been entered correctly by comparing the data presented in the systematic review versus information provided in the study reports. A second review author (ET or $\mathrm{CH}$ ) would have then spot-checked study characteristics against the trial report to confirm accuracy. 


\section{Assessment of risk of bias in included studies}

Two review authors (AMCC and ET) planned to independently assess risk of bias for each study using the criteria outlined in the Cochrane Handbook for Systematic Reviews of Interventions (Higgins 2011). We would have resolved disagreements by discussion or by involving another review author $(\mathrm{CH})$. We then planned to assess the risk of bias according to the following domains.

1. Random sequence generation.

2. Allocation concealment.

3. Blinding of participants and personnel.

4. Blinding of outcome assessment.

5. Incomplete outcome data.

6. Selective outcome reporting.

7. Other bias.

We planned to grade each potential source of bias as high, low or unclear and provide this grading in the 'Risk of bias' table using a quote from the study report together with a justification for our judgement. We planned to summarise the 'Risk of bias' judgements across different studies for each of the domains listed. We would have considered blinding separately for different key outcomes when necessary (e.g. for unblinded outcome assessment, risk of bias for hospital admissions may be very different than for patientreported adherence). When information on risk of bias related to unpublished data or correspondence with a trialist, we would have noted this in the 'Risk of bias' table. We planned to take into account the risk of bias of the included studies when considering treatment effects for each outcome.

\section{Assesment of bias in conducting the systematic review}

We conducted the review according to the published protocol (McCullough 2014b).

\section{Measures of treatment effect}

We planned to analyse dichotomous data as odds ratios and continuous data as mean differences or standardised mean differences. We planned to enter presented data as a scale with a consistent direction of effect. We would have undertaken metaanalyses only when this was meaningful (i.e. when treatments, participants and the underlying clinical question were similar enough for pooling to make sense). We would have provided a narrative description of skewed data reported as medians and interquartile ranges. When multiple trial arms were reported in a single trial, we planned to include only the relevant arms. When two comparisons (e.g. intervention A versus control and intervention $B$ versus control) were combined in the same meta-analysis, we would have halved the control group to avoid double-counting.

\section{Unit of analysis issues}

For dichotomous data, we planned to report the proportion of participants contributing to each outcome in comparison with the total number randomly assigned. For continuous data, the mean difference based on change from baseline would have been preferred over the mean difference based on absolute values. The unit of analysis would be the person. For cluster-randomised trials, to avoid a unit of analysis error, sensitivity analysis would have occurred at the participant level and would have incorporated adjustment using the intra-class correlation coefficient (ICC). For cross-over studies, we planned to extract data from the first period (before cross-over), to avoid any cross-over effects.

\section{Dealing with missing data}

We planned to contact investigators or study sponsors to verify key study characteristics and to obtain missing numerical outcome data when possible (e.g. when a study was identified as an abstract only). If this was not possible and the missing data were thought to introduce serious bias, we would have explored the impact of including such studies in the overall assessment of results by performing a sensitivity analysis.

\section{Assessment of heterogeneity}

We planned to use the $\mathrm{I}^{2}$ statistic to measure heterogeneity among the trials in each analysis. If we identified moderate $\left(I^{2}=30 \%\right.$ to $60 \%$ ) or substantial heterogeneity $\left(I^{2}=50 \%\right.$ to $90 \%$ ) (Higgins 2011), we planned to report this and explore possible causes by prespecified subgroup analysis.

\section{Assessment of reporting biases}

If we were able to pool more than 10 trials, we planned to create and examine a funnel plot to explore possible small-study biases.

\section{Data synthesis}

If a meta-analysis was appropriate, we would have used a fixedeffect model. If heterogeneity could not have been explained by the prespecified subgroup and sensitivity analyses, we planned to perform a sensitivity analysis using the random-effects model. If a meta-analysis was not appropriate, we planned to conduct a narrative synthesis of included studies.

\section{'Summary of findings' table}

We planned to create a 'Summary of findings' table using the primary and secondary outcomes stated previously. We planned to use the five GRADE considerations (study limitations, consistency of effect, imprecision, indirectness and publication bias) to assess the quality of a body of evidence as it related to the studies that contributed data to the meta-analyses for the prespecified outcomes. We would have used methods and recommendations described in Section 8.5 and Chapter 12 of the Cochrane Handbook for Systematic Reviews of Interventions (Higgins 2011) and planned to use GRADEpro GDT software (GRADEpro GDT). We would have justified all decisions to downgrade or upgrade the quality of studies by using footnotes, and made comments to aid readers' understanding of the review when necessary.

\section{Subgroup analysis and investigation of heterogeneity}

We planned to carry out the following subgroup analyses.

1. Type of intervention delivered.

2. Healthcare professional who delivered intervention (physician versus non-physician led, e.g. nurses compared with physiotherapists, pharmacists and other professionals).

3. Clinical setting (hospital care versus community services).

4. Duration of intervention (one-off intervention compared with more than one intervention).

5. Disease status (acute versus stable participants).

We planned to use the following outcomes in subgroup analyses. 
1. Adherence to at least one aspect of treatment (medication, airway clearance, medical devices or physical activity) as measured by direct (e.g. electronic monitoring, directly observed therapy) or indirect methods (e.g. self report, prescription refill data).

2. Rate of, duration of or time to first pulmonary exacerbation of bronchiectasis, defined according to the investigators' definition.

We planned to use the formal test for subgroup interactions in Review Manager (RevMan 2014). When both acute and stable participants were included in a study, we planned to explore them by subgroup analysis only if the results were reported separately.

\section{Sensitivity analysis}

We planned to carry out the following sensitivity analyses.
1. Sensitivity analysis excluding studies with a high risk of bias based on the 'Risk of bias' assessment.

2. Studies with less than $80 \%$ follow-up.

\section{RE S U L T S}

\section{Description of studies}

\section{Results of the search}

The electronic searches retrieved 37 articles reporting 36 studies, including 2 publications reporting the same study (Caine 2002; Sharples 2002). After screening of titles and abstracts, one study (two articles: Caine 2002; Sharples 2002) was retrieved for fulltext review. We wanted to review the full text for Yang 2012. We contacted the author to seek a full text but did not receive a response. None of these studies met the inclusion criteria (Characteristics of included studies; Figure 1). 
Figure 1. Study flow diagram.

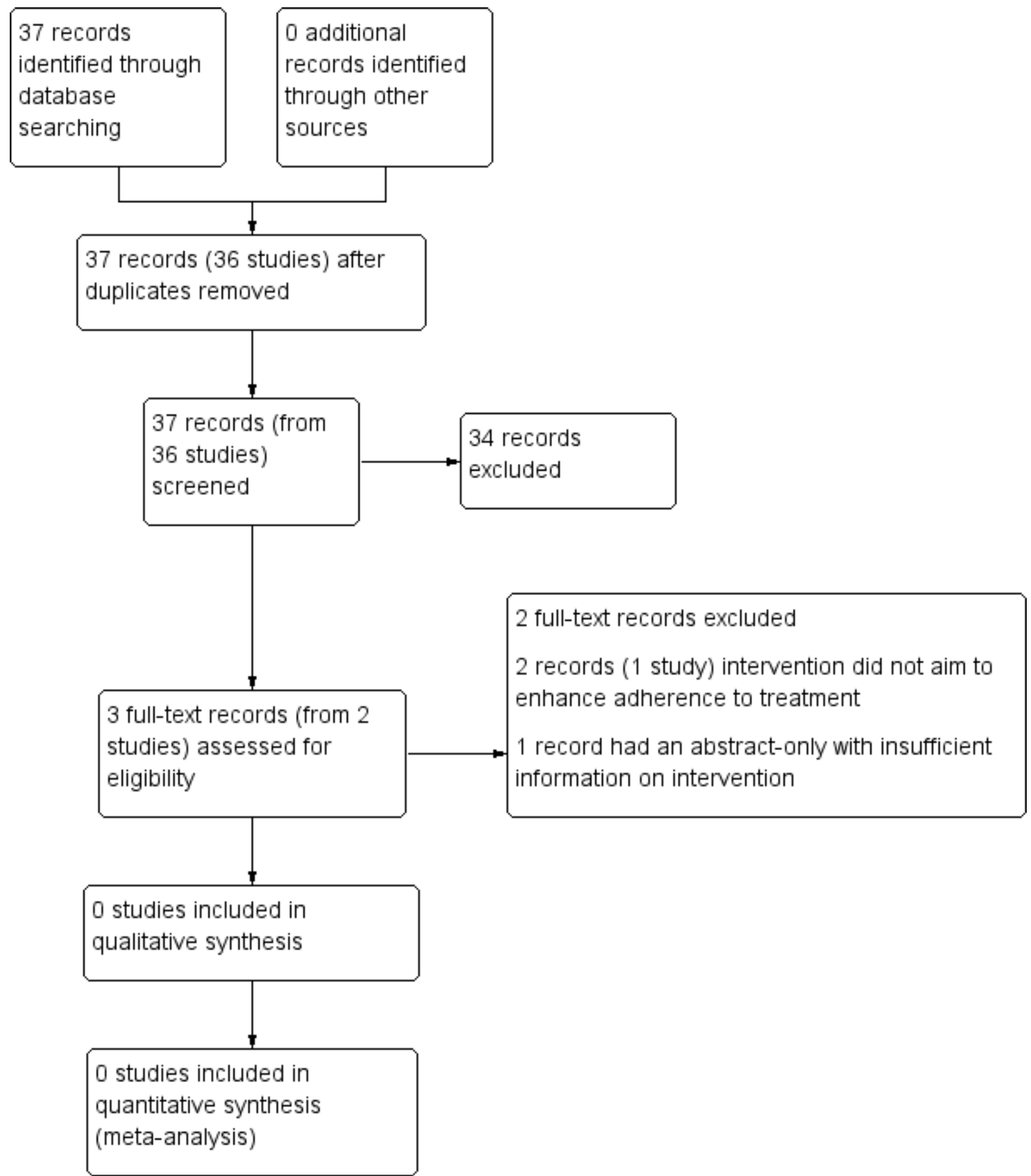

\section{Included studies}

No eligible studies were identified.

\section{Excluded studies}

We excluded one study that measured adherence (Caine 2002; Characteristics of excluded studies).
Caine 2002 tested nurse- versus doctor-led care with the aim of establishing equivalence in clinical outcomes including lung function and quality of life. The authors measured adherence to antibiotics as a secondary outcome and found that in a subgroup of participants using antibiotics, $100 \%(95 \% \mathrm{Cl} 89 \%$ to $100 \%)$ in the nurse-led intervention and $81 \%(95 \% \mathrm{Cl} 63 \%$ to $93 \%)$ in the doctor-led intervention reported being adherent to antibiotics $(\mathrm{n}=$ $31, P=0.02$ ). The authors did not report if there were any between- 
group differences in adherence to physiotherapy and inhalers. This study was excluded because it compared two different forms of treatment, nurse-led versus doctor-led care (much like an equivalence study for a new medication) and did not aim to enhance adherence.

\section{Studies awaiting classification}

We could not determine whether to include a further study (Yang 2012). We attempted to get access to a full text by contacting the authors but we did not receive a reply. Based on information in the abstract, they used a micro-blogging tool to encourage patients with bronchiectasis to keep a daily record of their disease symptoms, treatments and perceived treatment effects. Its primary aim appeared to be improved self-care. They also measured adherence to treatment but did not report it in the abstract. This study could not be included at this time because there was insufficient information in the abstract to determine if the intervention aimed to enhance adherence.

\section{Risk of bias in included studies}

No eligible studies were identified.

\section{Effects of interventions}

There are no data available as no eligible studies were identified.

\section{DISCUSSION}

\section{Summary of main results}

This review did not find any randomised controlled trials of interventions to enhance adherence in bronchiectasis. This finding is explained by a general lack of research into adherence in bronchiectasis. To date, research efforts have tended to focus on identifying new and effective treatments (De Soyza 2013), meaning that the effect of adherence to these treatments has only recently been described (Haworth 2013; McCullough 2014a). The effect of new treatments for bronchiectasis will be limited if adherence continues to be as low as recently reported (McCullough 2014a). As new treatments continue to develop, the ability to enhance adherence may become even more important.

\section{Overall completeness and applicability of evidence}

We did not include any studies in this review. We excluded one study (reported in two articles) that measured adherence. Caine 2002 did not test an intervention designed to enhance adherence. A further study (Yang 2012) is awaiting classification. The abstract did not report sufficient information about the intervention tested and the full publication could not be obtained from the authors.

\section{Quality of the evidence}

We did not include any studies in this review and could not assess their quality.

\section{Potential biases in the review process}

We completed a comprehensive search, without restrictions on language. However, we limited included studies to randomised control trials involving adults There may have been a greater range of interventions to examine if the review also incorporated other study designs or included children with bronchiectasis. We included any interventions aimed at enhancing adherence; however, this description is open to interpretation. Nieuwlaat 2014 included Caine 2002 in a Cochrane review of adherence interventions, whereas we have excluded it from this review.

\section{Agreements and disagreements with other studies or reviews}

We excluded a study by Caine 2002 as it did not aim to enhance adherence, in the same way that a study determining equivalence between one drug and another would not aim to enhance adherence. However, it was included in a recent systematic review of adherence interventions for any disease (Nieuwlaat 2014). This could be due to a very slight difference in the inclusion criteria, in which the authors of Nieuwlaat 2014 stated that they would include "interventions of any sort intended to affect adherence" (Nieuwlaat 2014). The study comparing nurse- verus doctor-led care will likely be included in a forthcoming update of a Cochrane review (French 2003). We also excluded studies comparing one treatment to another.

\section{AUTHORS' CONCLUSIONS}

\section{Implications for practice}

There are no studies determining the effects of interventions to enhance adherence to treatment in bronchiectasis. The lack of focus on interventions to enhance adherence reflects the general lack of emphasis that has been placed on this orphan disease: there is a lack of dedicated clinical funding (McCullough 2015; Welsh 2015) and specialist staff for bronchiectasis (McCullough 2015); managing adherence is not a priority for some clinicians (McCullough 2015); and others lack the skills and confidence to challenge patients about adherence (McCullough 2015). Without evidence-based interventions, clinicians do not know the most effective ways to change patient adherence behaviour. Research to determine effective interventions to enhance adherence is needed, so that clinicians can use these interventions to provide optimal care for their patients.

\section{Implications for research}

This comprehensive systematic review did not identify any interventions to enhance adherence in adults with bronchiectasis. An intervention to Change Adherence to treatment iN BronchiEtasis (CAN-BE) is currently being developed (McCullough 2015). This intervention has been theoretically derived and contains 12 behaviour change techniques, from which an individually-tailored intervention can be used with patients. This intervention is still in development, prior to feasibility and pilot testing. It is clear that a high-quality randomised controlled trial of an adherence intervention for adults with bronchiectasis is needed to determine its effects on adherence to treatment and clinical outcomes (pulmonary exacerbations, health-related quality of life and hospital admissions). Several challenges to this exist: separating intervention effects from a potential Hawthorne effect of being monitored as part of the study; developing an appropriate placebo intervention to attempt to blind participants and health professionals; and gaining adequate statistical power if cluster randomisation, or factorial designs are required.

It is likely that these trials will need to be conducted across several clinical centres to allow adequate patient recruitment. Infrastructure to support these types of studies is growing with the development of the Bronchiectasis Research and Academic 
Network in the United Kingdom (De Soyza 2013), the European Bronchiectasis Registry (EMBARC 2015) and the Bronchiectasis Research Registry in the United States (COPD Foundation 2015). However, the key challenge for research into this condition continues to be the lack of a dedicated research funding stream.

\section{ACKNOWLEDGEMENTS}

The review authors thank Emma Welsh, Managing Editor and Elizabeth Stovold, Trials Search Co-ordinator, Cochrane Airways Group, for their assistance with the planning of this protocol and the development of the review search strategy.
Sally Spencer was the editor for this protocol and commented critically on the review.

The background and methods section of this review is based on a standard template used by Cochrane Airways Group.

This project was supported by the National Institute for Health Research (NIHR), via Cochrane Infrastructure funding to the Cochrane Airways Group. The views and opinions expressed herein are those of the authors and do not necessarily reflect those of the Systematic Reviews Programme, NIHR, National Health Service (NHS) or the Department of Health. 


\section{RE F E R E N C E S}

\section{References to studies excluded from this review}

Caine 2002 \{published data only\}

Caine N, Sharples LD, Hollingworth W, French J, Keogan M, Exley A. A randomised controlled crossover trial of nurse practitioner versus doctor-led outpatient care in a bronchiectasis clinic. Thorax 2002;57:661-6.

Sharples LD, Edmunds J, Bilton D, Hollingworth W, Caine N, Keogan $\mathrm{M}$, et al. A randomised controlled crossover trial of nurse practitioner versus doctor led outpatient care in a bronchiectasis clinic. http://www.journalslibrary.nihr.ac.uk/ __data/assets/pdf_file/0004/64876/FullReport-hta6270.pdf (accessed 10 Nov 2015).

\section{References to studies awaiting assessment}

\section{Richmond 2016 \{published data only\}}

Richmond M, Lee Son N, Chilvers M, Mcllwaine M. Use of technology to measure adherence to, and quality of airway clearance techniques (ongoing). Pediatric Pulmonology 2016;51:371.

\section{Yang 2012 \{published data only\}}

Yang DW, Hu ZX, Song YL, Bai CX. A method to enhance cooperation and communication between patients and physicians at Zhongshan Hospital Fudan University: promoting self-care and communication for patients with bronchiectasis through daily diaries on Sina Weibo. 17th Congress of the Asian Pacific Society of Respirology; 2012 Dec 14-16; Hong Kong. 2012; Vol. 153:466.

\section{Additional references}

\section{Altenburg 2013}

Altenburg J, de Graaff CS, Stienstra Y, Sloos JH, van Haren EHJ, Koppers RJH, et al. Effect of azithromycin maintenance treatment on infectious exacerbations among patients with non-cystic fibrosis bronchiectasis. JAMA 2013;309(12):1251-9.

\section{Bilton 2013}

Bilton D, Daviskas E, Anderson SD, Kolbe J, King G, Stirling R, et al. A phase III randomised study of the efficacy and safety of inhaled dry powder mannitol for the symptomatic treatment of non-cystic fibrosis bronchiectasis. Chest 2013;144(1):215-25.

\section{Chang 2015}

Chang AB, Bell SC, Torzillo PJ, King PT, Maguire GP, Byrnes CA, et al. Chronic suppurative lung disease and bronchiectasis in children and adults in Australia and New Zealand. Medical Journal of Australia 2015;202(1):21-4.

\section{COPD Foundation 2015}

COPD Foundation. Bronchiectasis Research Registry. http:// www.copdfoundation.org/Research/Bronchiectasis-ResearchRegistry/Learn-More.aspx (accessed 12 August 2015).

\section{De Soyza 2013}

De Soyza A, Brown J, Loebinger M, on behalf of the Bronchiectasis Research and Academic Network. Research priorities in bronchiectasis. Thorax 2013;68:695-6.

\section{EMBARC 2015}

The European Bronchiectasis Registry. EMBARC. https:// www.bronchiectasis.eu/ (accessed 12 August 2015).

\section{Evans 2007}

Evans DJ, Bara A, Greenstone M. Prolonged antibiotics for purulent bronchiectasis in children and adults. Cochrane Database of Systematic Reviews 2007, Issue 2. [DOI: 10.1002/14651858.CD001392.pub2]

\section{Franco 2003}

Franco F, Sheikh A, Greenstone M. Short acting beta2-agonists for bronchiectasis. Cochrane Database of Systematic Reviews 2003, Issue 1. [DOI: 10.1002/14651858.CD003572]

\section{French 2003}

French J, Bilton D, Campbell F. Nurse specialist care for bronchiectasis. Cochrane Database of Systematic Reviews 2003 , Issue 7. [DOI: 10.1002/14651858.CD004359]

\section{Gibson 2013}

Gibson GJ, Loddenkemper R, Lundback B, Sibille Y. European Lung White Book. Sheffield: European Respiratory Society, 2013.

\section{Goyal 2014}

Goyal V, Chang AB. Combination inhaled corticosteroids and long-acting beta ${ }_{2}$-agonists for children and adults with bronchiectasis. Cochrane Database of Systematic Reviews 2014, Issue 6. [DOI: 10.1002/14651858.CD010327.pub2]

\section{GRADEpro GDT}

GRADEpro GDT: GRADEpro Guideline Development Tool [Software]. McMaster University, 2015 (developed by Evidence Prime, Inc.). http://www.guidelinedevelopment.org/ (accessed 10 November 2015)

\section{Hart 2014}

Hart A, Sugumar K, Milan SJ, Fowler SJ, Crossingham I. Inhaled hyperosmolar agents for bronchiectasis. Cochrane Database of Systematic Reviews 2014, Issue 5. [DOI: 10.1002/14651858.CD002996.pub3]

\section{Haworth 2013}

Haworth C, Foweraker J, Wilkinson P, Kenyon R, Bilton D. Multicenter randomized double blind placebo controlled trial of Promixin (colistin) delivered through the I-neb in patients with non-CF bronchiectasis and chronic Pseudomonas aeruginosa infection. American Journal of Respiratory and Critical Care Medicine 2013;187:A3511.

\section{Haynes 2008}

Haynes RB, Ackloo E, Sahota N, Mcdonald HP, Yao X. Interventions for enhancing medication adherence. 
Cochrane Database of Systematic Reviews 2008, Issue 2. [DOI: 10.1002/14651858.CD000011.pub3]

\section{Higgins 2011}

Higgins JPT, Green S (editors). Cochrane Handbook for Systematic Reviews of Interventions Version 5.1 [updated March 2011]. The Cochrane Collaboration, 2011. www.cochranehandbook.org.

\section{Lee 2013}

Lee AL, Burge A, Holland AE. Airway clearance techniques for bronchiectasis. Cochrane Database of Systematic Reviews 2013, Issue 5. [DOI: 10.1002/14651858.CD008351.pub2]

\section{McCullough 2014a}

McCullough AR, Hughes CM, Tunney MM, Elborn JS, Quittner AL, Bradley JM. Treatment adherence and health outcomes in patients with bronchiectasis infected with Pseudomonas aeruginosa. BMC Pulmonary Medicine 2014;14:107.

\section{McCullough 2015}

McCullough AR, Ryan C, O'Neill B, Bradley JB, Elborn JS, Hughes CM. Defining the content and delivery of an intervention to Change AdhereNce to treatment in BronchiEctasis (CAN-BE): a qualitative approach incorporating the Theoretical Domains Framework, behavioural change techniques and stakeholder expert panels. BMC Health Services Research 2015; Vol. 15:342.

\section{Nieuwlaat 2014}

Nieuwlaat R, Wilczynski N, Navarro T, Hobson N, Jeffery R, Keepanasseril A, et al. Interventions for enhancing medication adherence. Cochrane Database of Systematic Reviews 2014, Issue 11. [DOI: 10.1002/14651858.CD000011.pub4]

\section{Pasteur 2010}

Pasteur MC, Bilton D, Hill AT, British Thoracic Society Bronchiectasis non-CF Guideline Group. British Thoracic Society guideline for non-CF bronchiectasis. Thorax 2010;65(Suppl 1):i1-58.

\section{Pizzutto 2010}

Pizzuto SJ, Upham JW, Yerkovich ST, Chang AB. Inhaled nonsteroid anti-inflammatories for children and adults with bronchiectasis. Cochrane Database of Systematic Reviews 2010, Issue 4. [DOI: 10.1002/14651858.CD007525.pub2]

\section{RevMan 2014 [Computer program]}

The Nordic Cochrane Centre, The Cochrane Collaboration. Review Manager (RevMan). Version 5.3. Copenhagen: The Nordic Cochrane Centre, The Cochrane Collaboration, 2014.

\section{Seitz 2012}

Seitz A, Olivier KN, Adjemian J, Holland SM, Prevots DR. Trends in bronchiectasis among Medicare beneficiaries in the United States, 2000-2007. Chest 2012;142(2):432-9.

\section{CHARACTERISTICS OF STUDIES}

Characteristics of excluded studies [ordered by study ID]

\section{Serisier 2013}

Serisier DJ, Martin ML, McGuckin MA, Chen AC, Brain B, Biga S, et al. Effect of long-term, low-dose erythromycin on pulmonary exacerbations among patients with non-cystic fibrosis bronchiectasis. JAMA 2013;309(12):1260-7.

\section{Welsh 2015}

Welsh EJ, Evans DJ, Fowler SJ, Spencer S. Interventions for bronchiectasis: an overview of Cochrane systematic reviews. Cochrane Database of Systematic Reviews 2015, Issue 7. [DOI: 10.1002/14651858.CD010337.pub2]

\section{Weycker 2005}

Weycker D, Edelsberg J, Oster G, Tino G. Prevalence and economic burden of bronchiectasis. Clinical Pulmonary Medicine 2005;12(4):205-9.

\section{WHO 2003}

World Health Organisation. Adherence to long term therapies: evidence for action. http://www.who.int/chp/knowledge/ publications/adherence_report/en/ (accessed 9 October 2015) 2003.

\section{WHO 2007}

World Health Organisation. Global surveillance, prevention and control of chronic respiratory diseases: a comprehensive approach. http://www.who.int/entity/gard/publications/GARD \%20Book\%202007.pdf (accessed 14 November 2013) 2007.

\section{WHO 2010}

World Health Organisation. Global status report on noncommunicable diseases. http://whqlibdoc.who.int/ publications/2011/9789240686458_eng.pdf (accessed 14 November 2013) 2010.

\section{Wilkinson 2014}

Wilkinson M, Sugumar K, Milan SJ, Hart A, Crockett A, Crossingham I. Mucolytics for bronchiectasis. Cochrane Database of Systematic Reviews 2014, Issue 5. [DOI: 10.1002/14651858.CD001289.pub2]

\section{Wilson 2013}

Wilson R, Welte T, Polverino E, De Soyza A, Greville H, O'Donnell A, et al. Ciprofloxacin DPI in non-cystic fibrosis bronchiectasis: a phase II randomised study. European Respiratory Journal 2013;41(5):1107-5.

\section{References to other published versions of this review McCullough 2014b}

McCullough A, Ryan C, Bradley JM, O'Neill B, Elborn S, Hughes $C$. Interventions for enhancing adherence to treatment in adults with bronchiectasis. Cochrane Database of Systematic Reviews 2014, Issue 3. [DOI: 10.1002/14651858.CD011023] 


\section{Study}

Caine 2002

\section{Reason for exclusion}

Intervention did not aim to enhance adherence to treatment

Characteristics of studies awaiting assessment [ordered by study ID]

Richmond 2016

Methods

Participants

Interventions

Outcomes

Notes

Yang 2012

\begin{tabular}{ll}
\hline Methods & Randomized controlled trial \\
\hline Participants & 50 patients with bronchiectasis who visited a University Hospital \\
\hline Interventions & Intervention: Patients were trained to complete an online symptom diary using Sina Weibo \\
& Control: Typical care with outpatient services
\end{tabular}

Outcomes Compliance with treatment

Notes

\section{AP PEN DICES}

Appendix 1. Sources and search methods for the Cochrane Airways Group Specialised Register (CAGR)

Electronic searches: core databases

\begin{tabular}{ll}
\hline Database & Frequency of search \\
\hline CENTRAL (The Cochrane Library) & Monthly \\
\hline MEDLINE (Ovid) & Weekly \\
\hline EMBASE (Ovid) & Weekly \\
\hline PSyCINFO (Ovid) & Monthly \\
\hline CINAHL (EBSCO) & Monthly \\
\hline
\end{tabular}




\section{Handsearches: core respiratory conference abstracts}

\begin{tabular}{ll}
\hline Conference & Years searched \\
\hline American Academy of Allergy, Asthma and Immunology (AAAAI) & 2001 onwards \\
\hline American Thoracic Society (ATS) & 2001 onwards \\
\hline Asia Pacific Society of Respirology (APSR) & 2004 onwards \\
\hline British Thoracic Society Winter Meeting (BTS) & 2000 onwards \\
\hline Chest Meeting & 2003 onwards \\
\hline European Respiratory Society (ERS) & $1992,1994,2000$ onwards \\
\hline International Primary Care Respiratory Group Congress (IPCRG) & 2002 onwards \\
\hline Thoracic Society of Australia and New Zealand (TSANZ) & 1999 onwards \\
\hline
\end{tabular}

\section{MEDLINE search strategy used to identify trials for the CAGR}

\section{Bronchiectasis search}

1. exp Bronchiectasis/

2. bronchiect\$.mp.

3. bronchoect\$.mp.

4. kartagener\$.mp.

5. (ciliary adj3 dyskinesia).mp.

6. (bronchial\$ adj3 dilat\$).mp.

7. or/1-6

\section{Filter to identify RCTs}

1. exp "clinical trial [publication type]"/

2. (randomised or randomised).ab,ti.

3. placebo.ab,ti.

4. dt.fs.

5. randomly.ab,ti.

6. trial.ab,ti. 
7. groups.ab,ti.

8. or/1-7

9. Animals/

10. Humans/

11. 9 not (9 and 10)

12. 8 not 11

The MEDLINE strategy and RCT filter are adapted to identify trials in other electronic databases.

\section{Appendix 2. Search strategy to identify relevant trials from the CAGR}

\#1 BRONCH:MISC1

\#2 MeSH DESCRIPTOR Bronchiectasis Explode All

\#3 bronchiect*

\#4 \#1 or \#2 or \#3

\#5 MeSH DESCRIPTOR Medication Adherence

\#6 MeSH DESCRIPTOR Patient Acceptance of Health Care Explode All

\#7 MeSH DESCRIPTOR Patient Dropouts

\#8 complian* or noncomplian* or non-complian*

\#9 adhere $^{\star}$ or nonadhere ${ }^{\star}$ or non-adhere*

$\# 10$ persist $^{\star}$

\#11 refusal or refuse*

\#12 concord $^{*}$

\#13 co-operate*

\#14 conform*

\#15 accept*

\#16 comply*

$\# 17 \# 5$ or \#6 or \#7 or \#8 or \#9 or \#10 or \#11 or \#12 or \#13 or \#14 or \#15 or \#16

\#18 \#4 and \#17

[In search line \#1, MISC1 denotes the field in which the reference record has been coded for condition, in this case, bronchiectasis]

\section{WHAT'S NEW}

\begin{tabular}{lll}
\hline Date & Event & Description \\
\hline 7 July 2017 & Amended & $\begin{array}{l}\text { New literature search run to assess the need to update this } \\
\text { review. One potentially eligible ongoing study identified and } \\
\text { added to Studies awaiting classification. }\end{array}$ \\
\hline
\end{tabular}




\section{CONTRIBUTIONS OF AUTHORS}

All authors contributed to the protocol design, with the exception of ET. AMCC and ET screened titles, abstracts and full text articles. AMcC and ET prepared the first draft of the review. All review authors contributed to drafting of the review, gave final approval of the version to be published and are accountable for all aspects of the work.

\section{DECLARATIONS OF INTEREST}

AMcC is funded by the National Health and Medical Research Council (Australia) for work unrelated to this review (grant number 1044904).

\section{SOURCES OF SUPPORT}

\section{Internal sources}

- No sources of support supplied

\section{External sources}

- Public Health Agency HSC Research \& Development Division, Australia.

Cochrane Fellowship Award to Amanda McCullough providing funding for 2 days per week for 10 months to complete this review.

\section{DIFFERENCES BETWEEN PROTOCOL AND REVIEW}

We added the following to methods for future updates of this review: for cross-over studies, we planned to extract data from the first period (prior to cross-over), to avoid any cross-over effects.

\section{IN DEX TERMS}

\section{Medical Subject Headings (MeSH)}

Bronchiectasis [therapy]; Patient Compliance [*psychology]

\section{MeSH check words}

Adult; Humans 